\title{
Immunological nomograms predicting prognosis and guiding adjuvant chemotherapy in stage II colorectal cancer
}

This article was published in the following Dove Press journal: Cancer Management and Research

\author{
Yang Feng ${ }^{1, *}$ \\ Yaqi $\mathrm{Li}^{1,2, *}$ \\ Sanjun Cai ${ }^{1,2}$ \\ Junjie Peng ${ }^{1,2}$ \\ 'Department of Colorectal Surgery, \\ Fudan University Shanghai Cancer \\ Center, Shanghai 200032, People's \\ Republic of China; ${ }^{2}$ Department of \\ Oncology, Shanghai Medical College, \\ Fudan University, Shanghai 200032, \\ People's Republic of China
}

*These authors contributed equally to this work
Correspondence: Junjie Peng; Sanjun Cai Department of Colorectal Surgery, Fudan University Shanghai Cancer Center

Department of Oncology, Shanghai Medical College, Fudan University, 270 Dong'an Road, Shanghai 200032, People's Republic of China

Tel +86 I 80I 73I 7I22;

$+8613901815189$

Fax +862154175590

Email pengji67@hotmail.com;

caisanjun_sh@163.com
Background: The type, abundance, and location of tumor-infiltrating lymphocytes (TILs) have been associated with prognosis in colorectal cancer (CRC). This study was conducted to assess the prognostic role of TILs and develop a nomogram for accurate prognostication of stage II CRC.

Methods: Immunohistochemistry was conducted to assess the densities of intraepithelial and stromal CD3+, CD8+, CD45RO+, and FOXP3+ TILs, and to estimate PD-L1 expression in tumor cells for 168 patients with stage II CRC. The prognostic roles of these features were evaluated using COX regression model, and nomograms were established to stratify patients into low- and high-risk groups and compare the benefit from adjuvant chemotherapy.

Results: In univariate analysis, patients with high intraepithelial or stromal CD3+, CD8+, CD45RO+ and FOXP3+ TILs were associated significantly with better relapse-free survival (RFS) and overall survival (OS), except for stromal CD45RO+ TILs. In multivariate analysis, patients with high intraepithelial CD3+ and stromal FOXP3 + TILs were associated with better RFS $(p<0.001$ and $p=0.032$, respectively), while only stromal FOXP3+ TILs was an independent prognostic factor for OS $(p=0.031)$. The nomograms were well calibrated and showed a c-index of 0.751 and 0.757 for RFS and OS, respectively. After stratifying into low- and high-risk groups, the high-risk group exhibited a better OS from adjuvant chemotherapy (3-year OS of $81.9 \%$ vs $34.3 \%, p=0.006$ ).

Conclusion: These results may help improve the prognostication of stage II CRC and identify a high-risk subset of patients who appeared to benefit from adjuvant chemotherapy. Keywords: CD3, CD8, FOXP3, stage II, adjuvant chemotherapy

\section{Introduction}

5-fluorouracil-based adjuvant chemotherapy has been well established for patients with stage III colorectal cancer (CRC), but in stage II CRC, adjuvant chemotherapy is still hotly disputed considering the cost, toxicity, and limited survival benefit. ${ }^{1-4}$ A number of clinicopathological features (poor histological differentiation, T4 stage, $<12$ nodes harvested, high preoperative carcinoembryonic antigen (CEA) level, intestinal obstruction or perforation, and the presence of lymphovascular or perineural invasion) have been identified assisting the decision for adjuvant chemotherapy in stage II disease. ${ }^{1,5,6}$ However, only T4 stage has been proven to help identify a specific subset of stage II CRC patients who could achieve survival benefit from adjuvant chemotherapy. ${ }^{7}$ Besides, some polygene signatures have been widely explored, ${ }^{8,9}$ but there is still a long way to put these results into clinical 
practice. Identifying novel biomarkers to filter out the high-risk group of stage II CRC which could benefit from adjuvant chemotherapy is badly needed.

Adaptive immune response has been proven to influence the biological behavior of tumor cells, and the immune microenvironment formed by the type, abundance, and location of immune cells within tumor tissues were found to be a better predictor of patient survival than traditional clinicopathological features. ${ }^{10}$ Naito et al ${ }^{11}$ first demonstrated that the infiltration of tumor nests by CD8+ T-cells was a novel prognostic factor contributing to a better survival in CRC. Thereafter, CD3+ tumor-infiltrating lymphocytes (TILs) have been identified to be associated with favorable prognosis and a lower risk of metachronous metastasis in CRC. ${ }^{12,13}$ CD45RO+ TILs have also been reported to have prognostic significance. Pages et $\mathrm{al}^{14}$ revealed that high levels of CD45RO+ TILs were correlated with the absence of signs of early metastatic invasion, a less advanced pathological stage, and increased survival. In early-stage CRC, patients with a strong infiltration of CD45RO+ T-cells exhibited an increased expression of T-helper 1 and cytotoxicityrelated genes and helped predict tumor recurrence and survival. ${ }^{15}$ Regulatory T-cells engage in the maintenance of immunological self-tolerance by actively suppressing selfreactive lymphocytes. ${ }^{16,17}$ Nuclear transcription factor FOXP3, as a key regulatory gene for the development of regulatory T-cells, has been proven to be associated with improved survival in CRC. ${ }^{18}$ Therapeutic antibodies targeting the programmed cell death 1 protein (PD-1) and the programmed death-ligand 1 protein (PD-L1) have been proven to be effective in a number of cancer types. ${ }^{19,20} \mathrm{Li}$ et $\mathrm{al}^{21}$ revealed higher expressions of PD-1 and PD-L1 correlated with better prognosis of CRC patients. The objective of the current study was to assess and compare the prognostic role of PD-L1 and different types of TILs in stage II CRC and construct a nomogram for better prognostication, and to identify the subgroup of stage II CRC patients who can actually benefit from chemotherapy.

\section{Methods}

\section{Study group}

We 1:1 matched 84 recurrent stage II CRC patients to patients without recurrence, rendering 168 patients for analysis in our study. CRC tissue blocks were sent for next-generation sequencing (NGS) at Burning Rock Dx Corporation, Shanghai. No patients received preoperative therapy before radical surgery. Patients did not tolerate adequate course of adjuvant chemotherapy was excluded. All patients were regularly followed-up with a median follow-up time at 54.4 months (range 11.3-95.8 months). Informed consent had been obtained and this study was approved by the institutional review board of the Fudan University Shanghai Cancer Center.

\section{Immunohistochemistry (IHC)}

Immunohistochemically staining was performed according to standard protocol. Briefly, paraffin-embedded samples were cut into $4 \mu \mathrm{m}$ sections and placed on polylysinecoated slides. Paraffin sections were baked overnight at $58^{\circ} \mathrm{C}$, dewaxed in xylene, rehydrated through a graded series of ethanol, quenched for endogenous peroxidase activity in $0.3 \%$ hydrogen peroxide for 15 mins. Antigen retrieval was performed by high-pressure cooking in citrate buffer $(\mathrm{pH}=6.0)$ for about $20 \mathrm{mins}$, then allowed to cool to room temperature, blocking the nonspecific antibody binding sites in 5\% normal goat serum for 2 hrs. Sections were incubated at $37^{\circ} \mathrm{C}$ for $1.5 \mathrm{hrs}$ with rabbit polyclonal antibody against CD3 (1:400, Abcam, ab16669, USA), CD8 (1:400, Cell Signaling Technology, 70306S, USA), CD45RO (1:400, Dako, DK-2600 Glostrup, Denmark), FOXP3 (1:400, Abcam, ab20034, USA), and PD-L1 (1:100, Abcam, ab205921), in a moist chamber. Biotinylated secondary antibody was performed using the EnVision+System-HRP (AEC) (K4005, Dako, Glostrup, Denmark). Subsequently, sections were counterstained with hematoxylin (Sigma-Aldrich, St Louis, MO, USA). TMA slides were scanned by an automated scanning microscope and counted by Image-Pro Plus software (IPP; produced by Media Cybernetics Corporation, USA). Epithelial and stromal areas were calculated separately. Five independent visual fields (at $\times 400$ magnification), representing the most abundant lymphocytic infiltrates, were selected for each patient sample, and we used the mean density to stratify variables into dichotomous data for statistical analysis. PD-L1 expression score was the sum of the cytoplasmic and membrane scores. $^{22}$ Cytoplasmic expression level was scored as 0 (negative), 1 (weak), 2 (moderate) or 3 (strong), and membrane expression level was scored as 0 (absent) or 1 (present). PD-L1 scores 2/3/4 were counted as high, scores $0 / 1$ as low.

\section{Statistical analysis}

We used chi-square tests or Fisher's exact test to compare immunological biomarkers expression levels. Univariate and 
multivariate analyses were conducted using the Cox regression model. Nomograms were established by R software and the model performance for predicting outcome was evaluated by Harrell's concordance index (c-index). X-tile 3.6.1 software $^{23}$ (Yale University, New Haven, CT, USA) was used to determine the optimal cutoff values, stratifying the patients into low- and high-risk groups. Kaplan-Meier curves were drawn and log-rank tests were used to compare the survival data between different groups. $p$-values were accepted at $<0.05$ and all analyses were performed with the R 2.15.3 software.

\section{Results}

\section{Immunohistochemical characteristics}

Epithelial and in stromal TILs were evaluated separately. Utilizing tissue microarray (TMA), we quantified CD3+, CD8+, CD45RO+, and FOXP3+ cells by automatic imaging analysis on 168 stage II CRC samples. Representative immunohistochemical findings are demonstrated in Figure 1. Densities of each T-cell subset (cells $/ \mathrm{mm}^{2}$ ) were distributed as follows: intraepithelial CD3+ (mean 84; range 0-352), stromal CD3+ (mean 376; range 0-1380), intraepithelial CD8+ (mean 60; range 0-344), stromal CD8+ (mean 220; range 0-1120), intraepithelial CD45RO+ (mean 76; range 0 384), stromal CD45RO+ (mean 344; range 0-1600), intraepithelial FOXP3+ (mean 16; range 0-132), and stromal FOXP3+ (mean 132; range 0-600). Seventy-two patients were identified as PD-L1 low, and 96 patients were identified as PD-L1 high.

\section{Correlation of immune biomarkers with clinicopathological and molecular features}

Molecular features were available in 129 patients who successfully underwent NGS. As shown in Table 1, patients with high intraepithelial $\mathrm{CD} 3+, \mathrm{CD} 45 \mathrm{RO}+$, and stromal FOXP3+ TILs had a significantly higher incidence of normal preoperative CEA ( $p=0.010$, 0.013 , and 0.017 , respectively). Patients with high intraepithelial FOXP3+ TILs underwent less adjuvant chemotherapy $(p=0.019)$. More colon disease was observed in patients with high intraepithelial CD8+ TILs. Patients with high intraepithelial CD45RO+ and stromal CD8+ TILs had a significantly lower incidence of neural invasion ( $p=0.043$ and 0.046 , respectively). More T4 tumors were found in patients with high intraepithelial CD8+ TILs $(p=0.025)$. Patients with high intraepithelial CD45RO+ TILs had a significantly higher incidence of adequate lymph nodes harvested $(p=0.005)$. Patients with high intraepithelial $\mathrm{CD} 8+$ and $\mathrm{CD} 45 \mathrm{RO}+$ TILs had a significantly higher incidence of MSI-high ( $p=0.017$ and 0.002 , respectively). More ERBB2 mutation were observed in patients with high intraepithelial $\mathrm{CD} 45 \mathrm{RO}+$, FOXP3+, and stromal CD45RO+ TILs ( $p=0.019,0.020$, and 0.012 , respectively). More TP53 mutation were found in patients with high intraepithelial CD8+ and CD45RO+ TILs $(p=0.034$ and 0.025 , respectively). No significant differences were observed for gender, age, histology type, grade, vascular invasion, $A P C$ mutation, BRAF mutation, KRAS mutation, NRAS mutation, POLE mutation, PIK3CA mutation, and PTEN mutation.

\section{Prognostic factors}

In univariate analysis (Table 2), for tumor features, CEA was significantly associated with better relapse-free survival (RFS) and overall survival (OS) $(p<0.001$ and $p=0.015$, respectively). Number of lymph nodes harvested (LNH) were significantly associated with better OS $(p=0.012)$. Grade reached marginal significance for both RFS and OS ( $p=0.055$ and $p=0.068$, respectively). For molecular features, $B R A F$ and PTEN mutation were found to be significantly associated with better OS ( $p=0.007$ and $p=0.034$, respectively), whereas $B R A F$ mutation only reached marginal significance for RFS $(p=0.081)$. For Immune biomarkers, high intraepithelial or stromal $\mathrm{CD} 3+, \mathrm{CD} 8+, \mathrm{CD} 45 \mathrm{RO}+, \mathrm{FOXP} 3+$ TILs were significantly associated with better RFS and OS (all $p<0.05)$, except for high stromal CD45RO+ TILs $(p=0.110)$. PD-L1 was not associated with RFS or OS ( $p=0.574$ and $p=0.820$, respectively). A multivariate model was developed to test independent prognostic factors for RFS and OS (Table 3). In the first model (Model A, $n=168$ ), only tumor features and immune biomarkers with a $p<0.100$ in univariate analysis were included. CEA ( $p=0.040$; RR, 1.591; 95\% CI, 1.022-2.495), intraepithelial CD3+ TILs $(p<0.001 ; \mathrm{RR}, 0.192 ; 95 \%$ CI, 0.094 0.395 ), and stromal FOXP3+ TILs ( $p=0.032$; RR, 0.526; 95\% CI, 0.292-0.974) were found to be the strongest prognostic factors for RFS, whereas LNH $(p=0.010$; RR, 0.374; 95\% CI, 0178-0.784) and stromal FOXP3+ TILs ( $p=0.031$; RR, $0.249 ; 95 \% \mathrm{CI}, 0.071-0.878$ ) were proven to be independent prognostic factors for OS. The second model added molecular features (Model B, $n=129$ ) for analysis, intraepithelial CD3+ $(p<0.001 ; \mathrm{RR}, 0.179 ; 95 \%$ CI, 0.082-0.391) and stromal FOXP3+ TILs $(p=0.015$; 

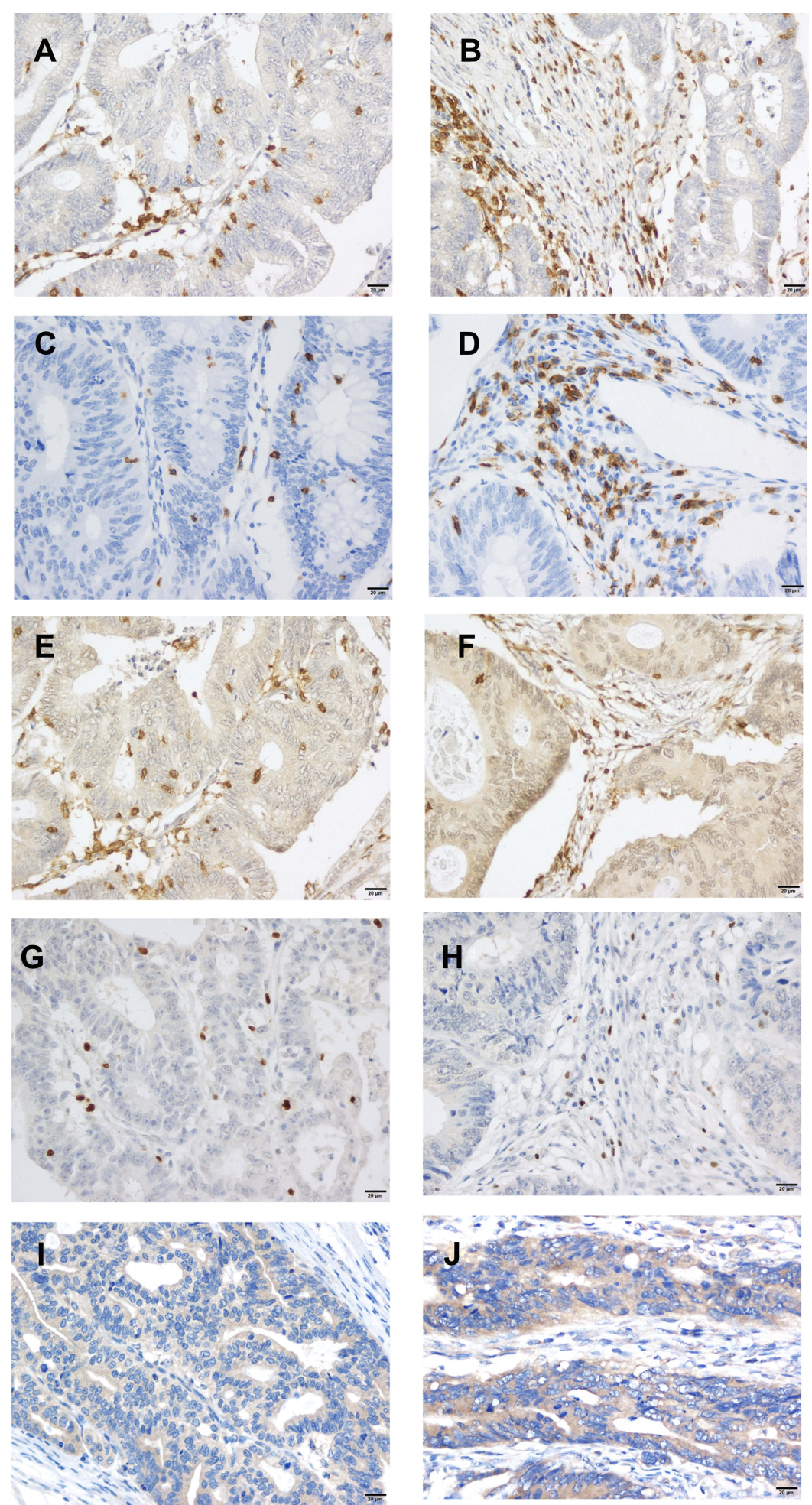

Figure I Representative examples of immunohistochemical findings for CD3, CD8, CD45RO, FOXP3, and PD-LI (original magnification, $\times$ 400). (A,B) Positive for intraepithelial and stromal CD3; (C,D) positive for intraepithelial and stromal CD8; (E,F) positive for intraepithelial and stromal CD45RO; (G,H) positive for intraepithelial and stromal FOXP3; (I,J) positive for cytoplasmic and membranous PD-LI.

RR, 0.425; 95\% CI, 0.214-0.845) retained significance for RFS. While for OS, stromal FOXP3+ TILs $(p=0.016$; RR, 0.155; 95\% CI, 0.034-0.703), LNH ( $p=0.038$; RR, 0.436;
95\% CI, 0.199-0.956), and PTEN mutation ( $p=0.001$; RR, 6.526 ; 95\% CI, 2.149-19.815) were the strongest prognostic factors. 


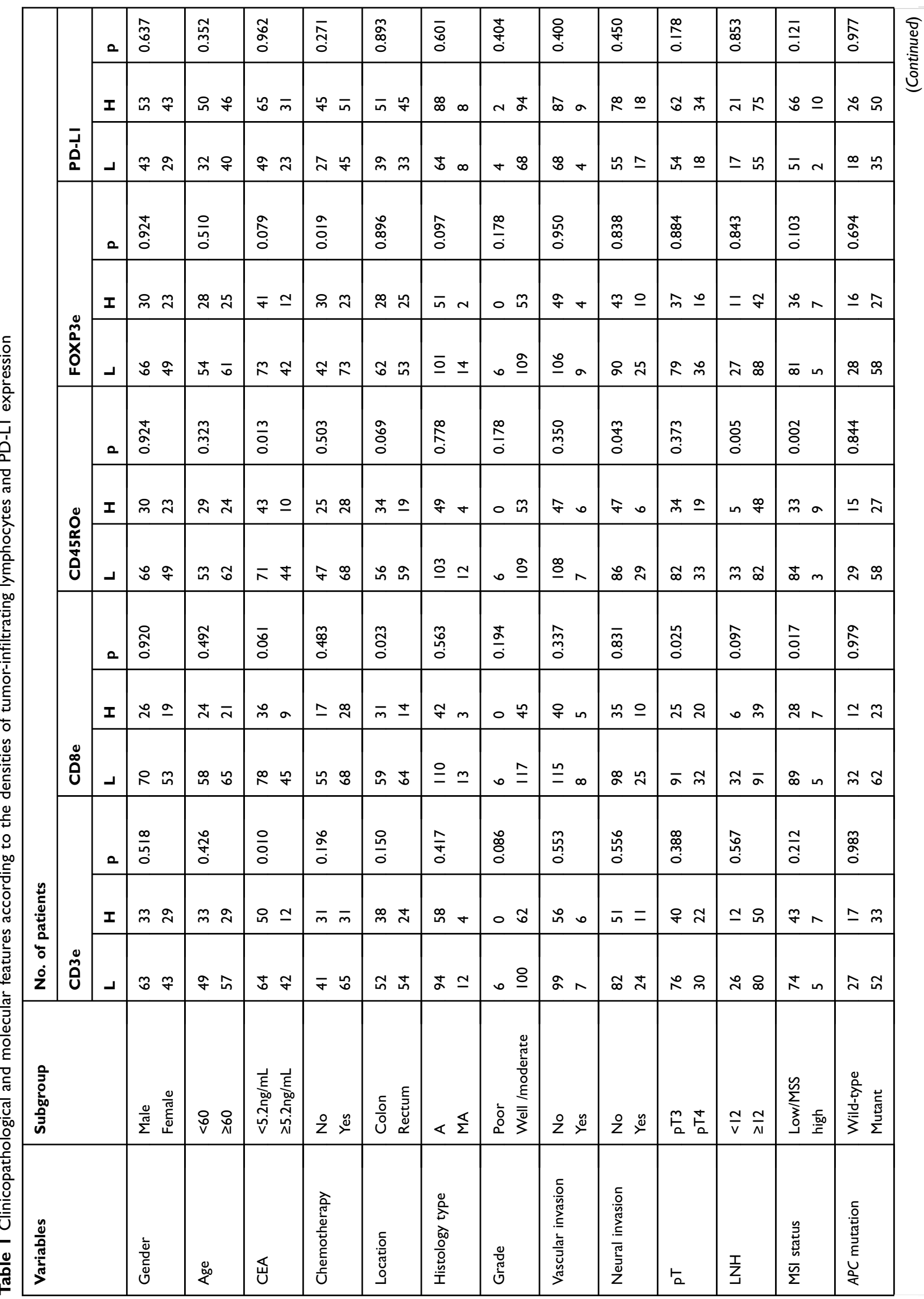




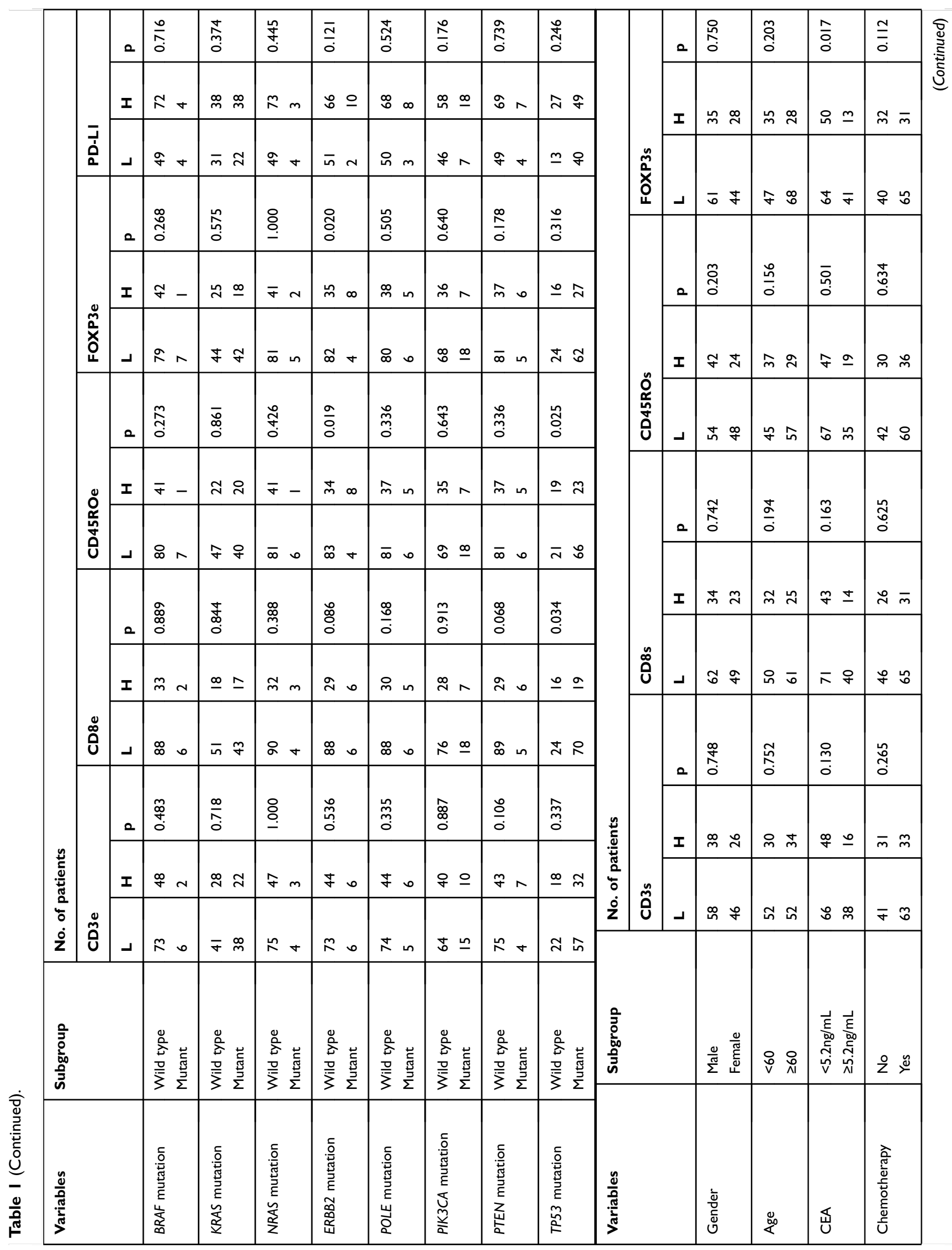




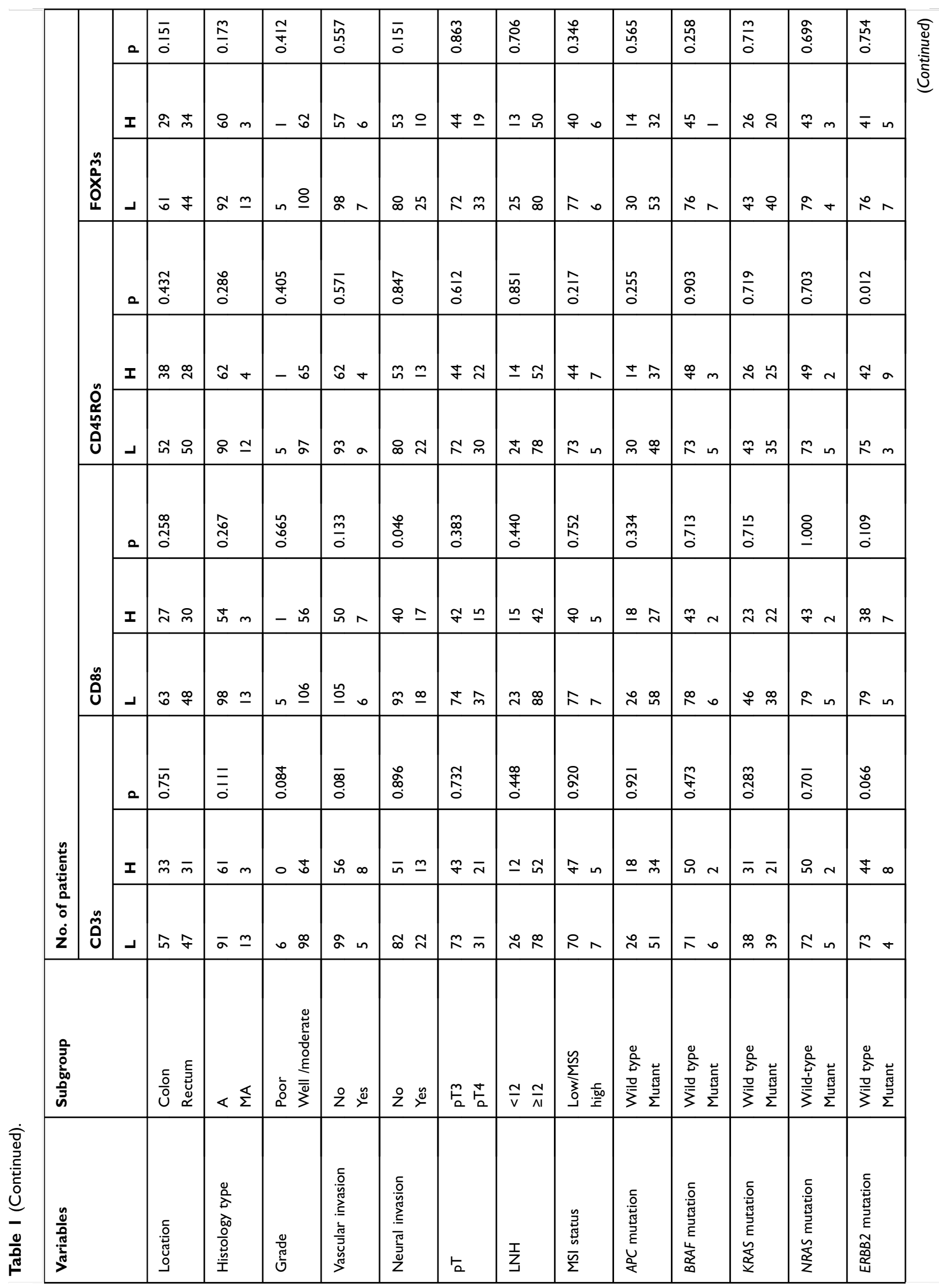




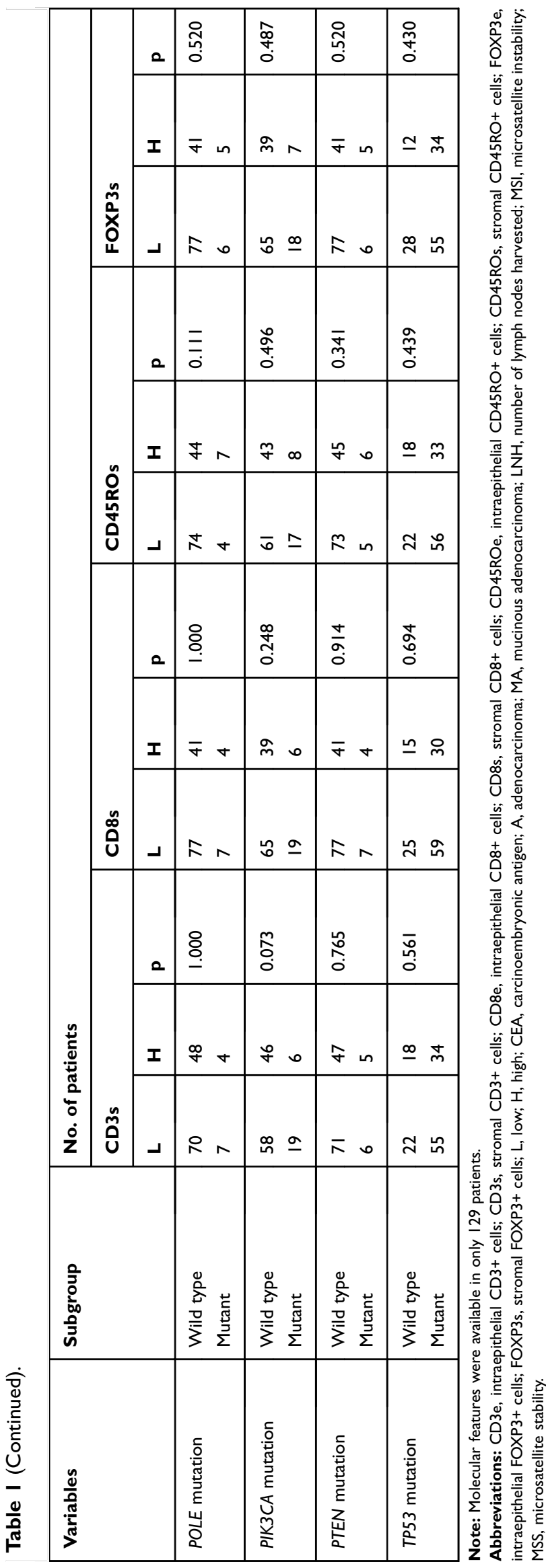

Nomogram construction, risk group stratification, and benefit from adjuvant chemotherapy

Variables with a $p$-value $<0.10$ in the multivariate analysis were included in nomogram construction. Three nomograms were constructed based on variables for RFS (nomogram A) and OS (nomogram B) in Model A and variables for OS (nomogram C) in Model B (see Figure 2), we did not establish a nomogram for RFS in Model B due to limited variables in the final model. Calibration curves were exhibited in Figure S1. For Model A, the nomograms were well calibrated and showed a c-index of 0.751 and 0.757 for RFS and OS, respectively. For Model B, the nomogram for OS was well calibrated and reached a cindex of 0.768 . X-tile software was used to select the optimal cutoff values. After stratifying into low- and high-risk groups (Figure S2), for nomogram A, high-risk patients had a significantly worse RFS low-risk patients (5-year RFS, $16.1 \%$ vs $58.2 \%, p<0.001$ ). For nomogram B and nomogram $\mathrm{C}$, worse OS was observed in high-risk group compared with low-risk group (5-year OS, $60.5 \%$ vs $90.6 \%, p<0.001 ; 5$-year OS, $45.0 \%$ vs $87.7 \%, p<0.001$, respectively). The relationship between risk groups and benefit from adjuvant chemotherapy is illustrated in Figure 3. No significant differences for RFS were observed between chemo-treated and chemo-naïve patients in different risk groups ( $p=0.625$ and 0.434 , respectively). For nomogram B, in high-risk group, chemo-treated patients had a better OS versus chemo-naïve patients, which reached marginal significance (5-year OS, 71.1\% vs $34.8 \%, p=0.105$ ). For nomogram $\mathrm{C}$, better OS was observed in chemo-treated patients compared with chemo-naïve patients (3-year OS, $81.9 \%$ vs $34.3 \%$, $p=0.006$ ).

\section{Discussion}

The therapeutic success of 5-fluorouracil-based adjuvant chemotherapy has been validated in stage III CRC, but not for patients with stage II disease. ${ }^{24,25}$ Up to now, only one nomogram predicting recurrence in stage II CRC has been constructed in literature by Hoshino et $\mathrm{al}^{26}$ which included sex, carcinoembryonic antigen, tumor location, tumor depth, lymphatic invasion, venous invasion, and number of lymph nodes studied, rendering a c-index of 0.64 . In our study, we first introduced immune biomarkers into nomogram construction, achieving a c-index of overwhelming 
Table 2 Univariate analyses of factors associated with relapse-free and overall survival

\begin{tabular}{|c|c|c|c|c|c|c|}
\hline \multirow[t]{2}{*}{ Variables } & \multicolumn{3}{|l|}{ RFS } & \multicolumn{3}{|l|}{ OS } \\
\hline & HR & $95 \% \mathrm{Cl}$ & $\mathbf{p}$ & HR & $95 \% \mathrm{Cl}$ & $\mathbf{p}$ \\
\hline \multicolumn{7}{|l|}{ Tumor features } \\
\hline Gender, female vs male & 0.829 & $0.534-1.287$ & 0.742 & 1.371 & $0.66 \mathrm{I}-2.843$ & 0.396 \\
\hline Age, $\geq 60$ vs $<60$ & 1.258 & $0.814-1.942$ & 0.301 & 1.679 & $0.793-3.554$ & 0.176 \\
\hline CEA, $\geq 5.2 \mathrm{ng} / \mathrm{mL}$ vs $<5.2 \mathrm{ng} / \mathrm{mL}$ & 2.274 & $1.472-3.515$ & $<0.001$ & 2.468 & I. $189-5.122$ & 0.015 \\
\hline Adjuvant chemotherapy, yes vs no & 1.118 & $0.722-1.732$ & 0.618 & 0.825 & $0.396-1.716$ & 0.606 \\
\hline Location, rectum vs colon & 1.335 & $0.867-2.054$ & 0.189 & 1.188 & $0.573-2.462$ & 0.643 \\
\hline Histology type, MA vs A & 0.827 & $0.38 \mathrm{I}-1.795$ & 0.631 & 0.654 & $0.155-2.754$ & 0.563 \\
\hline Grade, well/moderate vs poor & 0.411 & $0.166-1.021$ & 0.055 & 0.328 & $0.099-1.085$ & 0.068 \\
\hline Vascular invasion, yes vs no & 0.780 & $0.340-1.79 \mid$ & 0.558 & 0.773 & $0.183-3.256$ & 0.726 \\
\hline Neural invasion, yes vs no & 0.934 & $0.548-1.592$ & 0.802 & 0.403 & $0.122-1.332$ & 0.136 \\
\hline pT, T4 vs T3 & 0.993 & $0.62 \mathrm{I}-\mathrm{I} .587$ & 0.976 & 1.065 & $0.485-2.340$ & 0.876 \\
\hline $\mathrm{LNH}, \geq 12$ vs $<12$ & 0.756 & $0.464-|.23|$ & 0.261 & 0.389 & $0.186-0.085$ & 0.012 \\
\hline \multicolumn{7}{|l|}{ Molecular features } \\
\hline MSI status, high vs low/MSS & 0.770 & $0.310-1.915$ & 0.574 & 0.699 & $0.165-2.962$ & 0.627 \\
\hline APC mutation, $M$ vs $W T$ & 0.988 & $0.593-0.645$ & 0.962 & 2.173 & $0.819-5.765$ & 0.119 \\
\hline BRAF mutation, $M$ vs WT & 2.111 & $0.912-4.888$ & 0.081 & 4.399 & $1.507-12.842$ & 0.007 \\
\hline KRAS mutation, $\mathrm{M}$ vs $\mathrm{WT}$ & 1.110 & $0.687-1.792$ & 0.671 & 0.870 & $0.399-1.894$ & 0.725 \\
\hline NRAS mutation, $M$ vs WT & 0.795 & $0.250-2.531$ & 0.698 & 0.045 & $0.000-71.101$ & 0.410 \\
\hline ERBB2 mutation, $M$ vs WT & 0.833 & $0.335-2.074$ & 0.695 & 0.326 & $0.044-2.410$ & 0.272 \\
\hline POLE mutation, $M$ vs WT & 0.994 & $0.430-2.299$ & 0.988 & 1.531 & $0.523-4.480$ & 0.437 \\
\hline PIK3CA mutation, $M$ vs WT & 0.663 & $0.338-1.298$ & 0.231 & 0.862 & $0.325-2.287$ & 0.765 \\
\hline PTEN mutation, M vs WT & 1.061 & $0.459-2.456$ & 0.889 & 2.873 & $1.080-7.640$ & 0.034 \\
\hline TP53 mutation, M vs WT & 1.187 & $0.698-2.019$ & 0.527 & 1.173 & $0.493-2.792$ & 0.718 \\
\hline \multicolumn{7}{|l|}{ Immune biomarkers, high vs low } \\
\hline CD3e & 0.132 & $0.066-0.265$ & $<0.001$ & 0.276 & $0.105-0.726$ & 0.009 \\
\hline CD8e & 0.210 & $0.101-0.437$ & $<0.001$ & 0.253 & $0.076-0.835$ & 0.024 \\
\hline CD45ROe & 0.247 & $0.131-0.467$ & $<0.001$ & 0.287 & $0.100-0.825$ & 0.020 \\
\hline FOXP3e & 0.211 & $0.109-0.410$ & $<0.001$ & 0.195 & $0.059-0.644$ & 0.007 \\
\hline PD-LI & 1.134 & $0.73 I-1.76 \mid$ & 0.574 & 0.918 & $0.442-1.910$ & 0.820 \\
\hline CD3s & 0.375 & $0.224-0.638$ & $<0.001$ & 0.356 & $0.145-0.874$ & 0.024 \\
\hline CD8s & 0.361 & $0.209-0.623$ & $<0.001$ & 0.191 & $0.058-0.630$ & 0.007 \\
\hline CD45ROs & 0.497 & $0.307-0.805$ & 0.004 & 0.514 & $0.228-1.162$ & 0.110 \\
\hline FOXP3s & 0.257 & $0.148-0.444$ & $<0.001$ & 0.148 & $0.045-0.488$ & 0.002 \\
\hline
\end{tabular}

Note: Cox proportional hazards regression model, molecular features were available in only 129 patients.

Abbreviations: RFS, relapse-free survival; OS, overall survival; M, mutant; WT, wild type; CEA, carcinoembryonic antigen; A, adenocarcinoma; MA, mucinous adenocarcinoma; LNH, number of lymph nodes harvested; MSI, microsatellite instability; MSS, microsatellite stability; CD3e, intraepithelial CD3+ cells; CD3s, stromal CD3+ cells; CD8e, intraepithelial CD8+ cells; CD8s, stromal CD8+ cells; CD45ROe, intraepithelial CD45RO+ cells; CD45ROs, stromal CD45RO+ cells; FOXP3e, intraepithelial FOXP3+ cells; FOXP3s, stromal FOXP3+ cells.

0.751 and 0.757 for RFS and OS, respectively. Besides, the risk classification based on nomogram could identify a special high-risk subset of stage II CRC patients who may benefit from adjuvant chemotherapy.

Accumulating evidence suggests that effector/cytotoxic T-cells $\left(\mathrm{CD} 3+{ }^{12,13}\right.$ and $\left.\mathrm{CD} 8+{ }^{11,27}\right)$, memory T-cells $\left(\mathrm{CD} 45 \mathrm{RO}+{ }^{14,15}\right)$, and regulatory T-cells $\left(\mathrm{FOXP} 3+{ }^{16,18}\right)$ play important roles in antitumor immune response. Thus, the specific subsets of these TILs are thought to be indicators of host immune response to tumor cells and might be a target for immunotherapy. ${ }^{28,29}$ In the current study, we utilized a digitized, high-resolution image analysis system to count the number of TILs, and the mean densities of T-cell subsets were comparable with previous studies $\left(\mathrm{CD} 3+{ }^{10,30} \mathrm{CD} 8+,{ }^{18,31} \mathrm{CD} 45 \mathrm{RO}+,{ }^{18,32}\right.$ and FOXP $\left.3+{ }^{30,31}\right)$. Previous studies have demonstrated the high density of $\mathrm{CD} 3+, \mathrm{CD} 8+, \mathrm{CD} 45 \mathrm{RO}+$, or FOXP3+ TILs with MSI-high. ${ }^{18,30,33,34}$ In the current study, high 
Table 3 Multivariate Cox proportional model for predictors of relapse-free and overall survival

\begin{tabular}{|c|c|c|c|c|c|c|c|}
\hline \multicolumn{4}{|l|}{ DFS } & \multicolumn{4}{|l|}{ OS } \\
\hline Prognostic features & HR & $95 \% \mathrm{Cl}$ & $\mathbf{p}$ & Prognostic features & HR & $95 \% \mathrm{Cl}$ & $\mathbf{p}$ \\
\hline \multicolumn{4}{|l|}{ Model A (N= 168) } & \multicolumn{4}{|l|}{ Model A $(\mathrm{N}=168)$} \\
\hline CEA, $\geq 5.2 \mathrm{ng} / \mathrm{mL}$ vs $<5.2 \mathrm{ng} / \mathrm{mL}$ & 1.591 & $1.022-2.475$ & 0.040 & CEA, $\geq 5.2 \mathrm{ng} / \mathrm{mL}$ vs $<5.2 \mathrm{ng} / \mathrm{mL}$ & 2.080 & $0.995-4.349$ & 0.052 \\
\hline CD3e, high vs low & 0.192 & $0.094-0.395$ & $<0.001$ & $\mathrm{LNH}, \geq 12$ vs $<12$ & 0.374 & $0.178-0.784$ & 0.010 \\
\hline CD8s, high vs low & 0.600 & $0.338-1.064$ & 0.080 & CD8s, high vs low & 0.325 & $0.093-1.143$ & 0.080 \\
\hline FOXP3s, high vs low & 0.526 & $0.292-0.974$ & 0.032 & FOXP3s, high vs low & 0.249 & $0.07 I-0.878$ & 0.031 \\
\hline \multicolumn{4}{|l|}{ Model B $(\mathrm{N}=129)$} & \multicolumn{4}{|l|}{ Model B (N=129) } \\
\hline CD3e, high vs low & 0.179 & $0.082-0.391$ & $<0.001$ & CD8e, high vs low & 0.282 & $0.067-1.178$ & 0.083 \\
\hline \multirow[t]{3}{*}{ FOXP3s, high vs low } & 0.425 & $0.214-0.845$ & 0.015 & FOXP3s, high vs low & 0.155 & $0.034-0.703$ & 0.016 \\
\hline & & & & $\mathrm{LNH}, \geq 12$ vs $<12$ & 0.436 & $0.199-0.956$ & 0.038 \\
\hline & & & & PTEN mutation, $M$ vs WT & 6.526 & $2.149-19.815$ & 0.001 \\
\hline
\end{tabular}

Notes: Cox proportional hazards regression model. Model $A$ included tumor features and immune biomarkers with a $p<0.10$ in univariate analysis ( $N=168)$. Model $B$ included tumor features, immune biomarkers, and molecular features with a $p<0.10$ in univariate analysis $(\mathrm{N}=129)$. $A$ backward $L R$ (likelihood ratio) elimination with a threshold of $p=0.10$ was presented in the final model.

Abbreviations: RFS, relapse-free survival; OS, overall survival; M, mutant; WT, wild type; CEA, carcinoembryonic antigen; LNH, number of lymph nodes harvested; CD3e, intraepithelial CD3+ cells; CD8e, intraepithelial CD8+ cells; CD8s, stromal CD8+ cells; FOXP3s, stromal FOXP3+ cells.

densities of CD45RO+ and CD8+ cells, but not that of CD3 + or FOXP3+ cells, are significantly associated with MSI-high. We used multivariate analysis to assess the prognostic roles of these immune biomarkers and found intraepithelial CD3+ TILs and stromal FOXP3+ TILs were the strongest prognostic factors for RFS, whereas only stromal FOXP3+ TILs were an independent prognostic factor for OS. Our study revealed patients with high intraepithelial CD3+ and stromal FOXP3+ TILs had a significantly higher incidence of normal preoperative CEA, which partially explained the good prognosis associated with these biomarkers. Although $\mathrm{Li}$ et $\mathrm{al}^{21}$ concluded PD-L1 correlated with better prognosis in CRC patients, our study did not prove the prognostic role PDL1, which is in agreement with Masugi's ${ }^{22}$ study.

Despite numerous studies have demonstrated the prognostic roles of immune-related biomarkers using IHC, seldom have these studies involved molecular features for analysis. In our study, 129 patients successfully underwent NGS and classic mutations for CRC were evaluated for their prognostic roles. KRAS mutation and PTEN mutation were found to be significant factors for OS in univariate analysis, while only PTEN mutation was demonstrated as an independent prognostic factor in multivariate analysis after adjusting for clinicopathological features and immune biomarkers. PTEN is a candidate tumor suppressor and key negative regulator of the PI3K pathway, involving in cell proliferation, migration, and survival. ${ }^{35}$ Somatic mutations in PTEN were detected in about $6 \%$ of sporadic CRC, and PTEN mutation was found to be associated with proximal tumors, mucinous histology, MSI-H, CIMP-high, and $B R A F$ mutation. ${ }^{36}$ In our study, $8.5 \%$ PTEN mutation was observed, $36.4 \%$ of MSI-high patients were observed in PTEN mutation group compared with $6.8 \%$ in the wild-type group, which is in consistence with previous studies. ${ }^{36,37}$ Recent reports suggest that PTEN exerts an important tumor suppressor role in colorectal carcinogenesis $^{35}$ and correlative analyses have associated loss of PTEN with poorer survival, ${ }^{38,39}$ which is in agreement with our study.

Our study is limited as a retrospective study in nature, further validations from other institutions are merited. Secondly, we did not separate colon and rectal cancer for further study due to limited sample size. Moreover, considering intratumoral heterogeneity, we admit that our study might still fall short of capturing heterogeneity within tumor. Despite of these shortcomings, this is the largest study elucidating the prognostic roles of the densities of various types of TILs focusing on stage II CRC, and we first used nomogram to visualize the results and stratify patients into low- and high-risk groups. More importantly, it is easier for clinical use than signatures or other risk classification systems. 
A

Points

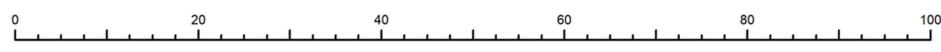

CD3e

CD8s

FOXP3s

CEA

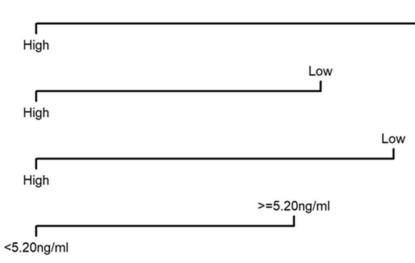

Total points

1-year survival

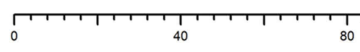

80

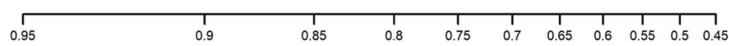

3-year survival

5-year survival

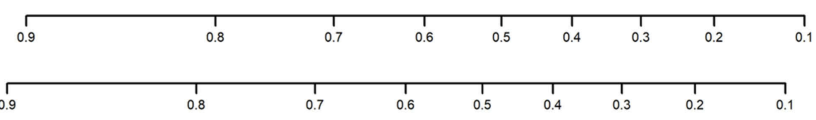

B

Points

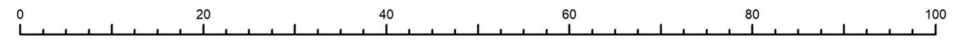

LNH

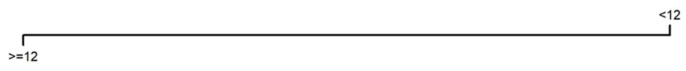

CD8s

FOXP3s

CEA

High
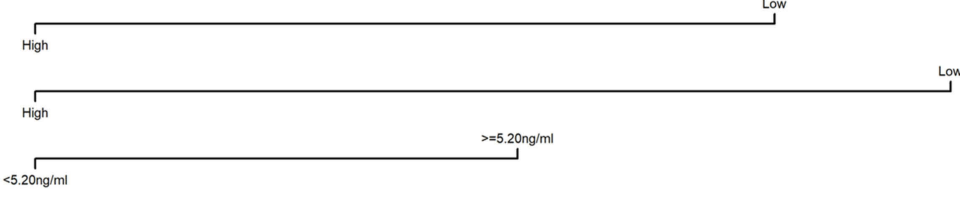

Total points

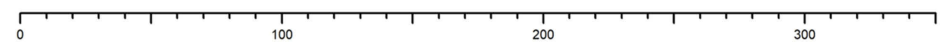

1-year survival

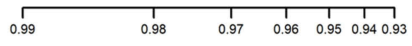

3-year survival

5-year survival

C

Points

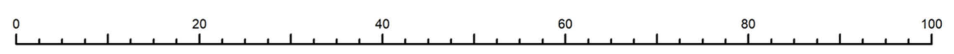

LNH

$<12$

CD8e

FOXP3s

PTEN

$$
>>12
$$
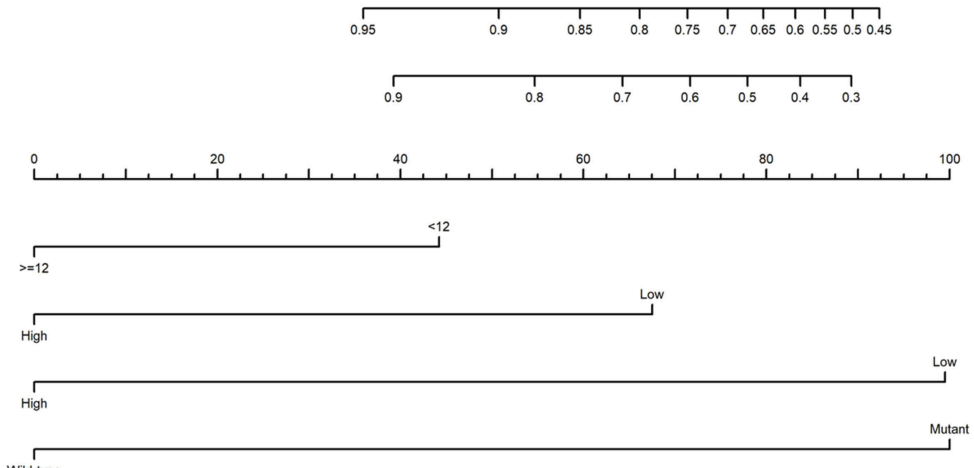

Total points

1-year survival

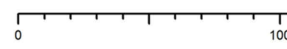

100
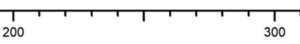

3-year survival

5-year survival

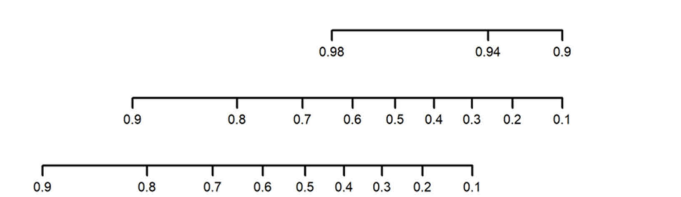

Figure 2 Nomograms for I-, 3-, and 5-year probabilities of survival. (A) Nomogram A predicting relapse-free survival based on Model A, with a c-index of 0.75 I; (B) nomogram B predicting overall survival based on Model A, with a c-index of 0.757 ; (C) nomogram $\mathrm{C}$ predicting overall survival based on Model B, with a c-index of 0.768 .

Abbreviations: CEA, carcinoembryonic antigen; LNH, number of lymph nodes harvested; CD3e, intraepithelial CD3+ cells; CD8s, stromal CD8+ cells; CD8e, intraepithelial CD8+ cells; FOXP3s, stromal FOXP3+ cells.

In summary, we constructed nomograms which may help

Furthermore, we identified a high-risk subset of stage II CRC

to predict RFS and OS in patients with stage II CRC. patients who appeared to benefit from adjuvant chemotherapy. 
A
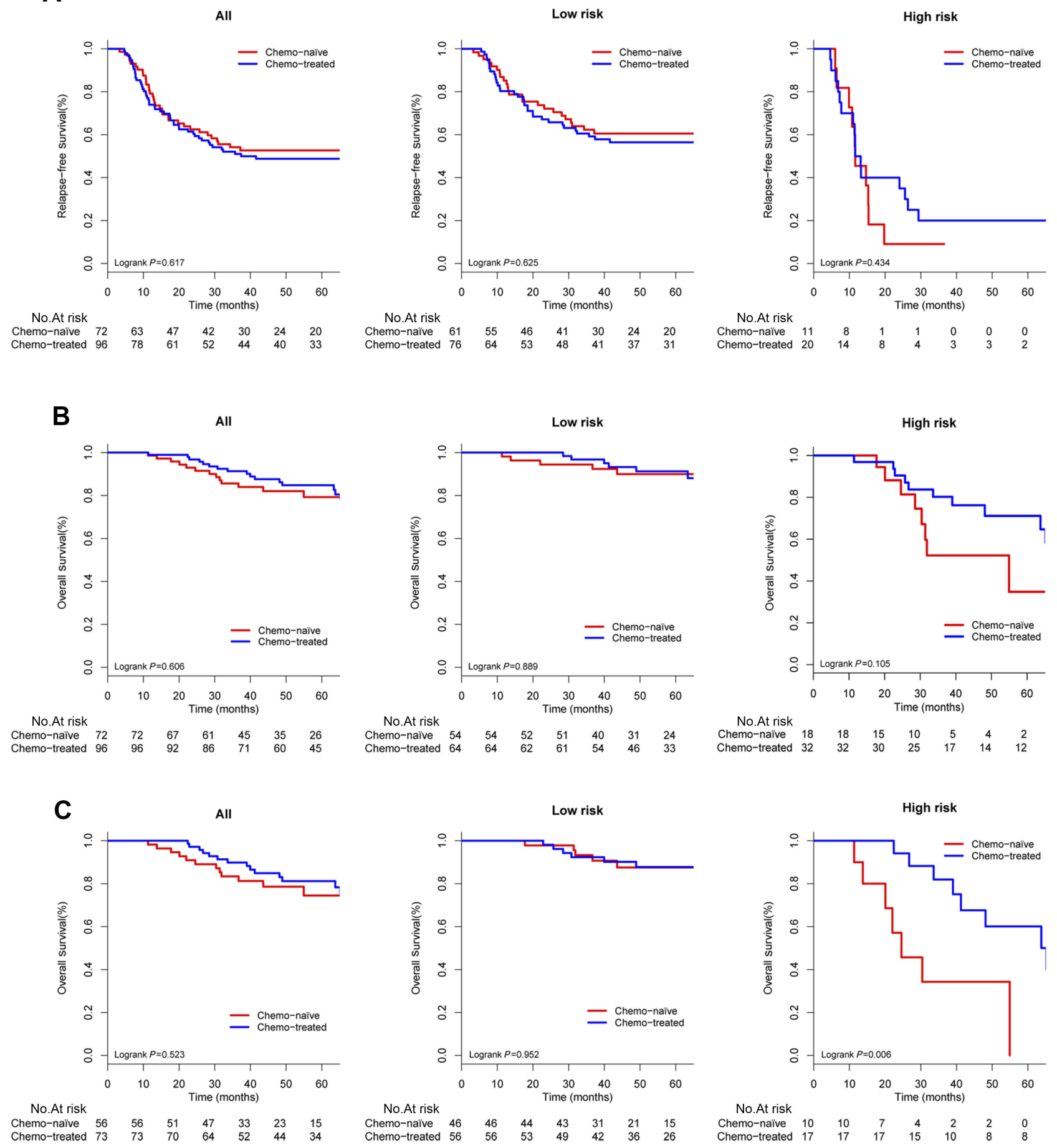

Figure 3 Relationship between risk groups and benefit from adjuvant chemotherapy in stage II colorectal cancer patients. (A) Relapse-free survival based on nomogram A classification; (B) overall survival based on nomogram B classification; (C) overall survival based on nomogram C classification.

\section{Ethics approval and consent to participate}

Informed consent had been obtained and this study was approved by the institutional review board of the Fudan
University Shanghai Cancer Center. The patient consent was written informed consent, and that this study was conducted in accordance with the Declaration of Helsinki. 


\section{Abbreviation list}

TILs, tumor-infiltrating lymphocytes; CRC, colorectal cancer; dMMR, deficient mismatch repair; pMMR, proficient mismatch repair; CEA, carcinoembryonic antigen; PD-1, programmed cell death 1 protein; PD-L1, programmed death-ligand 1 protein; NGS, next-generation sequencing; TMA, tissue microarray; RFS, relapse-free survival; OS, overall survival; LNH, lymph nodes harvested; NCCN, National Comprehensive Cancer Network; MSI, microsatellite instability; MSS, microsatellite stability; $\mathrm{CD} 3 \mathrm{e}$, intraepithelial CD3+ cells; CD3s, stromal CD3+ cells; CD8e, intraepithelial $\mathrm{CD} 8+$ cells; $\mathrm{CD} 8 \mathrm{~s}$, stromal $\mathrm{CD} 8+$ cells; CD45ROe, intraepithelial CD45RO+ cells; CD45ROs, stromal CD45RO+ cells; FOXP3e, intraepithelial FOXP3+ cells; FOXP3s, stromal FOXP3+ cells.

\section{Author contributions}

All authors contributed to data analysis, drafting or revising the article, gave final approval of the version to be published, and agree to be accountable for all aspects of the work.

\section{Disclosure}

The abstract for this paper was accepted as poster presentation at the 2018 ASCO conference. The authors report no other potential conflicts of interest in this work.

\section{References}

1. Kucukzeybek Y, Dirican A, Demir L, et al. Adjuvant chemotherapy and prognostic factors in stage II colon cancer-izmir oncology group study. Asian Pac J Cancer Prev. 2015;16:2413-2418. doi:10.7314/ apjcp.2015.16.6.2413

2. Benson AB 3rd, Schrag D, Somerfield MR, et al. American society of clinical oncology recommendations on adjuvant chemotherapy for stage II colon cancer. J Clin Oncol. 2004;22:3408-3419. doi:10.1200/JCO.2004.05.063

3. O'Connor ES, Greenblatt DY, LoConte NK, et al. Adjuvant chemotherapy for stage II colon cancer with poor prognostic features. $J$ Clin Oncol. 2011;29:3381-3388. doi:10.1200/JCO.2010.34.3426

4. Gray R, Barnwell J, McConkey C, Hills RK, Williams NS, Kerr DJ. Adjuvant chemotherapy versus observation in patients with colorectal cancer: a randomised study. Lancet. 2007;370:2020-2029. doi:10.1016/S0140-6736(07)61866-2

5. Quah HM, Chou JF, Gonen M, et al. Identification of patients with high-risk stage II colon cancer for adjuvant therapy. Dis Colon Rectum. 2008;51:503-507. doi:10.1007/s10350-008-9246-z

6. Okada K, Sadahiro S, Suzuki T, et al. The size of retrieved lymph nodes correlates with the number of retrieved lymph nodes and is an independent prognostic factor in patients with stage II colon cancer. Int $J$ Colorectal Dis. 2015;30:1685-1693. doi:10.1007/s00384-015-2357-9

7. Kumar A, Kennecke HF, Renouf DJ, et al. Adjuvant chemotherapy use and outcomes of patients with high-risk versus low-risk stage II colon cancer. Cancer. 2015;121:527-534. doi:10.1002/cncr.29072
8. Gao S, Tibiche C, Zou J, et al. Identification and construction of combinatory cancer hallmark-based gene signature sets to predict recurrence and chemotherapy benefit in stage II colorectal cancer. JAMA Oncol. 2016;2:37-45. doi:10.1001/jamaoncol.2015.3413

9. Tian X, Zhu X, Yan T, et al. Recurrence-associated gene signature optimizes recurrence-free survival prediction of colorectal cancer. Mol Oncol. 2017;11:1544-1560. doi:10.1002/1878-0261.12117

10. Galon J, Costes A, Sanchez-Cabo F, et al. Type, density, and location of immune cells within human colorectal tumors predict clinical outcome. Science. 2006;313:1960-1964. doi:10.1126/science.1129139

11. Naito Y, Saito K, Shiiba K, et al. CD8+ T cells infiltrated within cancer cell nests as a prognostic factor in human colorectal cancer. Cancer Res. 1998;58:3491-3494.

12. Sinicrope FA, Rego RL, Ansell SM, Knutson KL, Foster NR, Sargent DJ. Intraepithelial effector $(\mathrm{CD} 3+)$ /regulatory (FoxP3+) T-cell ratio predicts a clinical outcome of human colon carcinoma. Gastroenterology. 2009;137:1270-1279. doi:10.1053/j.gastro.2009.06.053

13. Laghi L, Bianchi P, Miranda E, et al. CD3+ cells at the invasive margin of deeply invading (pT3-T4) colorectal cancer and risk of post-surgical metastasis: a longitudinal study. Lancet Oncol. 2009;10:877-884. doi:10.1016/S1470-2045(09)70186-X

14. Pages F, Berger A, Camus M, et al. Effector memory T cells, early metastasis, and survival in colorectal cancer. $N$ Engl $J$ Med. 2005;353:2654-2666. doi:10.1056/NEJMoa051424

15. Pages F, Kirilovsky A, Mlecnik B, et al. In situ cytotoxic and memory T cells predict outcome in patients with early-stage colorectal cancer. $J$ Clin Oncol. 2009;27:5944-5951. doi:10.1200/JCO.2008.19.6147

16. Fontenot JD, Gavin MA, Rudensky AY. Foxp3 programs the development and function of $\mathrm{CD} 4+\mathrm{CD} 25+$ regulatory $\mathrm{T}$ cells. Nat Immunol. 2003;4:330-336. doi:10.1038/ni904

17. Hori S, Nomura T, Sakaguchi S. Control of regulatory T cell development by the transcription factor Foxp3. Science. 2003;299:10571061. doi: $10.1126 /$ science. 1079490

18. Salama P, Phillips M, Grieu F, et al. Tumor-infiltrating FOXP3+ T regulatory cells show strong prognostic significance in colorectal cancer. $J$ Clin Oncol. 2009;27:186-192. doi:10.1200/JCO.2008.18.7229

19. Tumeh PC, Harview CL, Yearley JH, et al. PD-1 blockade induces responses by inhibiting adaptive immune resistance. Nature. 2014;515:568-571. doi:10.1038/nature13954

20. Le DT, Durham JN. Mismatch repair deficiency predicts response of solid tumors to PD-1 blockade. Science. 2017;357:409-413. doi:10.1126/science.aan6733

21. Li Y, Liang L, Dai W, et al. Prognostic impact of programed cell death-1 (PD-1) and PD-ligand 1 (PD-L1) expression in cancer cells and tumor infiltrating lymphocytes in colorectal cancer. Mol Cancer. 2016;15:55. doi:10.1186/s12943-016-0539-x

22. Masugi Y, Nishihara R, Yang J, et al. Tumour CD274 (PD-L1) expression and T cells in colorectal cancer. Gut. 2017;66:14631473. doi:10.1136/gutjnl-2016-311421

23. Camp RL, Dolled-Filhart M, Rimm DL. X-tile: a new bio-informatics tool for biomarker assessment and outcome-based cut-point optimization. Clin Cancer Res. 2004;10:7252-7259. doi:10.1158/10780432.CCR-04-0713

24. Kohne $\mathrm{CH}$. Should adjuvant chemotherapy become standard treatment for patients with stage II colon cancer? Against the proposal. Lancet Oncol. 2006;7:516-517.

25. Sobrero A. Should adjuvant chemotherapy become standard treatment for patients with stage II colon cancer? For the proposal. Lancet Oncol. 2006;7:515-516. doi:10.1016/S1470-2045(06)70727-6

26. Hoshino N, Hasegawa S, Hida K, et al. Nomogram for predicting recurrence in stage II colorectal cancer. Acta Oncol. 2016;55:14141417. doi:10.1080/0284186X.2016.1223881

27. Chiba T, Ohtani H, Mizoi T, et al. Intraepithelial CD8+ T-cell-count becomes a prognostic factor after a longer follow-up period in human colorectal carcinoma: possible association with suppression of micrometastasis. Br J Cancer. 2004;91:1711-1717. doi:10.1038/sj.bjc.6602201 
28. Zou W. Regulatory T cells, tumour immunity and immunotherapy. Nat Rev Immunol. 2006;6:295-307. doi:10.1038/nri1806

29. Disis ML, Bernhard H, Jaffee EM. Use of tumour-responsive T cells as cancer treatment. Lancet. 2009;373:673-683. doi:10.1016/S01406736(09)60404-9

30. Nosho K, Baba Y, Tanaka N, et al. Tumour-infiltrating T-cell subsets, molecular changes in colorectal cancer, and prognosis: cohort study and literature review. J Pathol. 2010;222:350-366. doi:10.1002/path.2774

31. Suzuki H, Chikazawa N, Tasaka T, et al. Intratumoral CD8(+) T/ FOXP3 $(+)$ cell ratio is a predictive marker for survival in patients with colorectal cancer. Cancer Immunol Immunother. 2010;59:653661. doi:10.1007/s00262-009-0781-9

32. Lee WS, Park S, Lee WY, Yun SH, Chun HK. Clinical impact of tumor-infiltrating lymphocytes for survival in stage II colon cancer. Cancer. 2010;116:5188-5199. doi:10.1002/cncr.25293

33. Guidoboni M, Gafa R, Viel A, et al. Microsatellite instability and high content of activated cytotoxic lymphocytes identify colon cancer patients with a favorable prognosis. Am J Pathol. 2001;159:297-304. doi:10.1016/S0002-9440(10)61695-1

34. Michel S, Benner A, Tariverdian M, et al. High density of FOXP3-positive $\mathrm{T}$ cells infiltrating colorectal cancers with microsatellite instability. $\mathrm{Br} \mathrm{J}$ Cancer. 2008;99:1867-1873. doi:10.1038/sj.bjc.6604756
35. Di Cristofano A, Pandolfi PP. The multiple roles of PTEN in tumor suppression. Cell. 2000;100:387-390. doi:10.1016/s0092-8674(00) 80674-1

36. Day FL, Jorissen RN, Lipton L, et al. PIK3CA and PTEN gene and exon mutation-specific clinicopathologic and molecular associations in colorectal cancer. Clin Cancer Res. 2013;19:3285-3296. doi:10.1158/1078-0432.CCR-12-3614

37. Parsons DW, Wang TL, Samuels Y, et al. Colorectal cancer: mutations in a signalling pathway. Nature. 2005;436:792. doi:10.1038/nature03934

38. Laurent-Puig P, Cayre A, Manceau G, et al. Analysis of PTEN, BRAF, and EGFR status in determining benefit from cetuximab therapy in wild-type KRAS metastatic colon cancer. J Clin Oncol. 2009;27:5924-5930. doi:10.1200/JCO.2008.21.6796

39. Sood A, McClain D, Maitra R, et al. PTEN gene expression and mutations in the PIK3CA gene as predictors of clinical benefit to antiepidermal growth factor receptor antibody therapy in patients with KRAS wild-type metastatic colorectal cancer. Clin Colorectal Cancer. 2012;11:143-150. doi:10.1016/j.clcc.2011.12.001 


\section{Supplementary material}

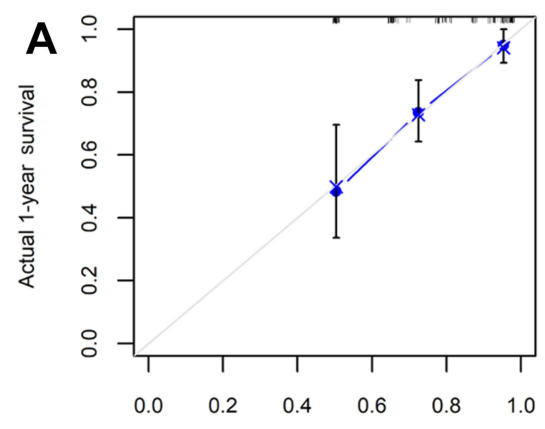

Nomogram predicted 1-year survival

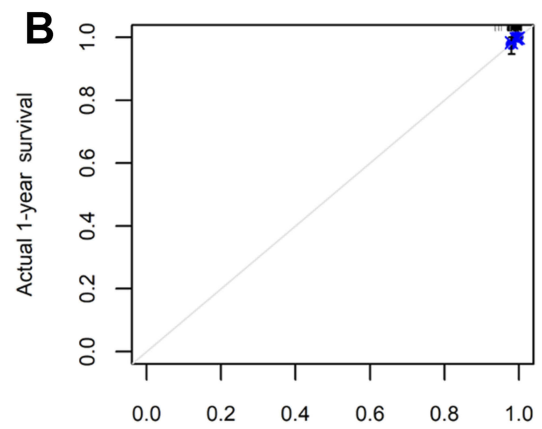

C

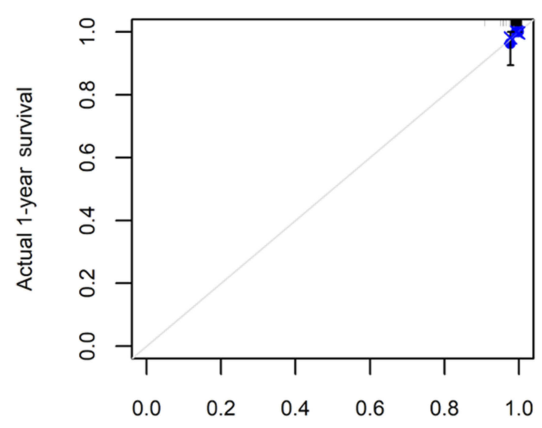

Nomogram predicted 1-year survival

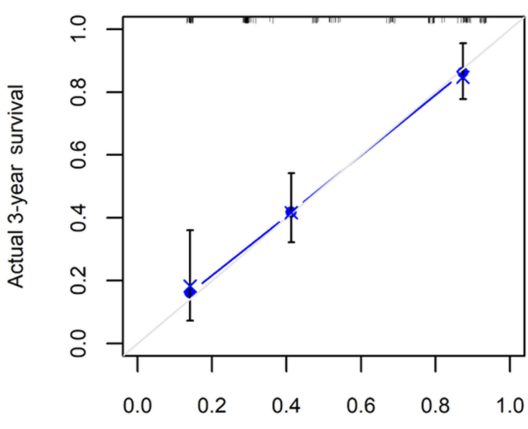

Nomogram predicted 3-year survival

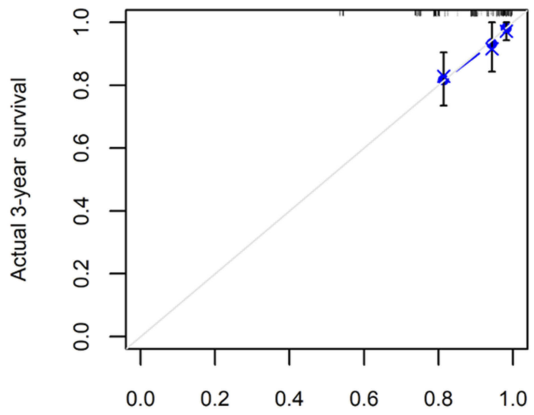

Nomogram predicted 3-year survival

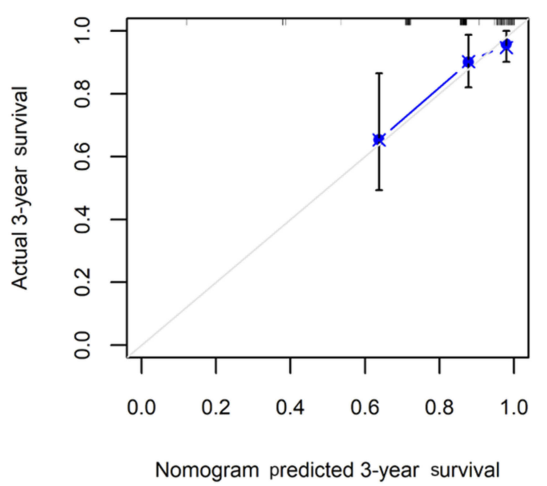

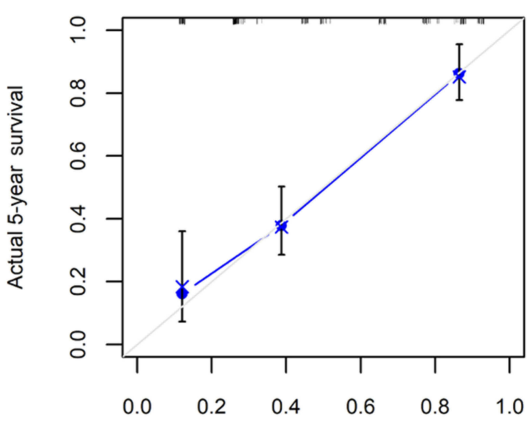

Nomogram predicted 5-year survival

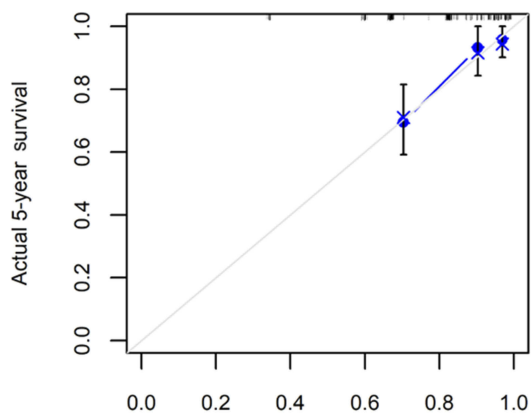

Nomogram predicted 5-year survival

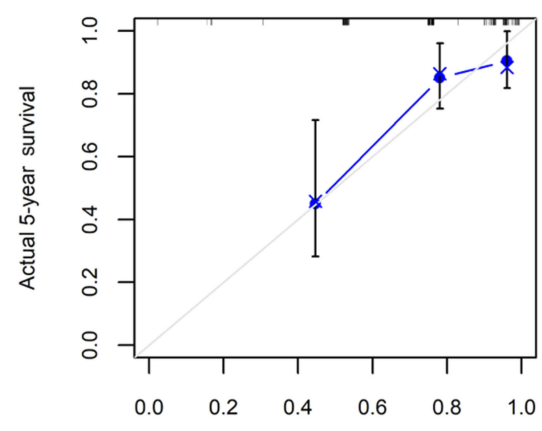

Nomogram predicted 5-year survival

Figure SI Calibration of the nomograms for I-, 3-, and 5-year probabilities of survival. The x-axis shows the nomogram-predicted survival at I, 3, and 5 years, and the $y$ axis shows the observed actual survival and $95 \%$ confidence intervals. (A) Calibration of nomogram A; (B) calibration of nomogram B; (C) calibration of nomogram C. 

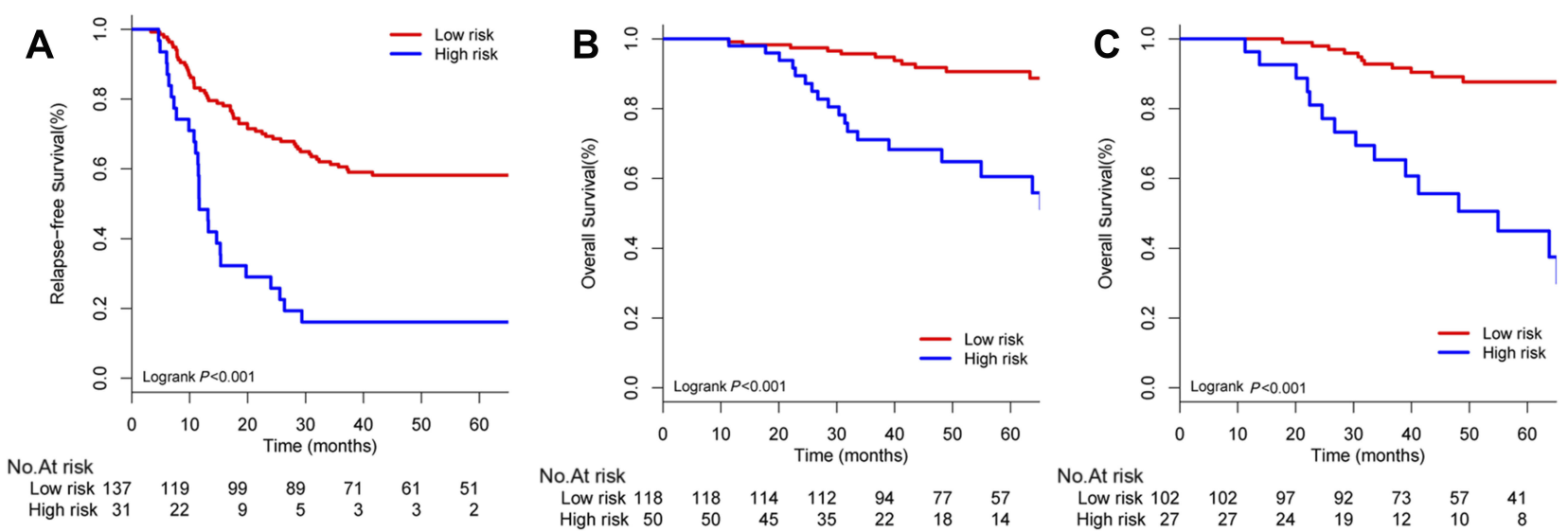

Figure S2 Survival curves comparing different risk groups. The patients were stratified into two groups according to the cutoff values generated by $X$-tile program. (A) Relapse-free survival based on nomogram A classification; (B) Overall survival based on nomogram B classification; (C) overall survival based on nomogram C classification.

\section{Publish your work in this journal}

Cancer Management and Research is an international, peer-reviewed open access journal focusing on cancer research and the optimal use of preventative and integrated treatment interventions to achieve improved outcomes, enhanced survival and quality of life for the cancer patient.
The manuscript management system is completely online and includes a very quick and fair peer-review system, which is all easy to use. Visit http://www.dovepress.com/testimonials.php to read real quotes from published authors. 